\title{
Quartz crystal microbalance coated with PEDOT-PSS/PVA nanofiber for a high-performance humidity sensor
}

\author{
Trisna Julian ${ }^{1}$, Aditya Rianjanu ${ }^{1}$, Shidiq Nur Hidayat ${ }^{1}$, Ahmad Kusumaatmaja ${ }^{1}$, Roto Roto ${ }^{2}$, and \\ Kuwat Triyana ${ }^{1,3}$ \\ ${ }^{1}$ Department of Physics, Faculty of Mathematics and Natural Sciences, Universitas Gadjah Mada, \\ Sekip Utara BLS 21, Yogyakarta 55281, Indonesia \\ ${ }^{2}$ Department of Chemistry, Faculty of Mathematics and Natural Sciences, Universitas Gadjah Mada, \\ Sekip Utara BLS 21, Yogyakarta 55281, Indonesia \\ ${ }^{3}$ Institute of Halal Industry and System (IHIS), Universitas Gadjah Mada, Sekip Utara, \\ Yogyakarta 55281, Indonesia
}

Correspondence: Kuwat Triyana (triyana@ugm.ac.id)

Received: 29 April 2019 - Revised: 24 June 2019 - Accepted: 26 June 2019 - Published: 16 July 2019

\begin{abstract}
Quartz crystal microbalance (QCM) coated with poly(3,4-ethylenedioxythiophene) and polystyrene sulfonate mixed with polyvinyl alcohol (PEDOT-PSS/PVA) nanofiber has been fabricated as a humidity sensor using the electrospinning method. Three types of PEDOT-PSS/PVA nanofiber sensors are fabricated with different needle-to-collector electrospinning distances. The scanning electron microscope images confirm the presence of beads in the nanofiber structure. The results show that the sensor mass deposition increased with the decrease in needle-to-collector distance. The best sensor performance is exhibited by the sample with medium needle-tocollector distance (QCM NF 2). The QCM NF 2 nanofiber sensor shows excellent sensitivity of up to $33.56 \mathrm{~Hz}$ per percentage point of relative humidity, with rapid response $(5.6 \mathrm{~s})$ and recovery $(3.5 \mathrm{~s})$ times, good linearity, excellent repeatability, low hysteresis, and long-term stability and response. The QCM PEDOT-PSS/PVA nanofiber sensor provides a simple method to fabricate high-performance humidity sensors.
\end{abstract}

\section{Introduction}

Recently, various types of humidity sensors have been proposed (Lensch et al., 2019; Xu et al., 2019; Zhang et al., 2018). A humidity sensor with high sensitivity, rapid response time, low hysteresis, and long-term stability has become the main goal to pursue (Haidry et al., 2015; Yao et al., 2017). To date, several sensing methods, including resistance, capacitance, optical fiber, and quartz crystal microbalance (QCM), have been used to develop a humidity sensor (Blank et al., 2016; Xu et al., 2019). A QCM is a kind of piezoelectric crystal resonator, of which the frequency resonance relies on the change of the loading mass. The QCM is often used to develop a gas or vapor sensor (Beißner et al., 2017; Li et al., 2019; Rianjanu et al., 2018d; Xu et al., 2018). Many researchers used the QCM to fabricate a high- performance humidity sensor (Jin et al., 2017; Nie et al., 2017). A humidity sensor based on the QCM provides realtime, accurate, and convenient detection.

The frequency resonance of an uncoated QCM is naturally influenced by the change of the humidity level. However, the response is relatively small and deficient to utilize as a humidity sensor. The performance of a humidity sensor based on the QCM can be increased by modifying the QCM surface using coating materials. Various sensing materials, such as polymers, metal oxides, and some functional materials, have been introduced to modify the QCM sensors (Pascariu et al., 2016; Yang et al., 2016; Zhang et al., 2018). Hygroscopic and hydrophilic materials have often been used because of their superior capability of absorbing water molecules (Chen et al., 2016). 
Poly(3,4-ethylenedioxythiophene):polystyrene sulfonate (PEDOT-PSS) is a kind of hydrophilic polymer that is often used in organic electronic devices (Muckley et al., 2017). The hydrophilic properties of PEDOT-PSS makes it a promising sensing material for a humidity sensor based on the QCM method. Several studies have reported the development of a humidity sensor using PEDOT-PSS as sensor active layer (Hossein-Babaei et al., 2019; Park et al., 2018; Taccola et al., 2013). Furthermore, the use of PEDOT-PSS polymer for developing a humidity sensor using a quartz crystal microbalance sensor platform was also available. Jaruwongrungsee et al. (2011) obtained a high-sensitivity humidity sensor using quartz crystal microbalance printed PEDOT-PSS (Jaruwongrungsee et al., 2011). Meanwhile, a composite of PEDOTPSS and polyvinyl alcohol (PVA) as an active layer of the QCM humidity sensor was also applied (Choi et al., 2015). Another work utilized PEDOT-PSS to fabricate a QCMbased multimodal humidity and pressure sensor (Muckley et al., 2016).

Various methods, including spin coating, electrospinning, drop casting, and self-assembled monolayer methods, have been utilized to coat a thin polymer film onto the QCM substrate (Rianjanu et al., 2018b, 2019; Triyana et al., 2018). Compared with those fabricated using other methods, electrospun nanofiber has the advantages of higher specific surface area (SSA) and porosity, which lead to a high performance (Adamyan et al., 2018; Choi et al., 2010). Electrospinning is a simple, continuous, and efficient process used to fabricate nanofiber films. The morphology of electrospun nanofiber depends on several parameters, including the needle-to-collector electrospinning distance, applied high voltage, and solution viscosity of the polymer solution (Rianjanu et al., 2018c; Rodoplu and Mutlu, 2012). When these parameters are maintained, the electrospinning method will produce a controllable nanofiber with high reproducibility. A nanofiber structure possesses a high surface area, which is beneficial to its utilization as a humidity sensor (Ding et al., 2009). Thus, the use of PEDOT-PSS mixed with polyvinyl alcohol (PEDOT-PSS/PVA)-nanofiber-coated QCM chips to develop a humidity sensor has considerable potential. In this study, we aim to develop a humidity sensor based on QCM chips using PEDOT-PSS/PVA to produce nanofiber as a sensing layer. Since PEDOT-PSS polymer cannot be electrospun independently due to its low viscosity, it is often mixed with a high-viscosity and water-soluble polymer. We used PVA to improve the electrospinning ability of the PEDOTPSS polymer, as has been done previously (Kumar et al., 2016; Cho et al., 2012; Liu et al., 2011).

\section{Materials and method}

PEDOT-PSS PH $1000(1.3 \%(w / w))$ dispersed in water was purchased from Heraeus C. Starck, Goslar, Germany. PVA with a molecular weight of $89000-98000 \mathrm{Da}$ was purchased
Table 1. Frequency shift and calculated coating load after nanofiber deposition.

\begin{tabular}{lrrr}
\hline Trial & $\begin{array}{r}\text { Electrospinning } \\
\text { distance }(\mathrm{cm})\end{array}$ & $\begin{array}{r}\text { Frequency } \\
\text { shift }(\mathrm{Hz})\end{array}$ & $\begin{array}{r}\text { Coating } \\
\text { load (ng) }\end{array}$ \\
\hline QCM NF 1 & 7.5 & 8200 & 1800 \\
QCM NF 2 & 10.0 & 3500 & 800 \\
QCM NF 3 & 12.5 & 1800 & 400 \\
\hline
\end{tabular}

from Sigma-Aldrich, St. Louis, MO, USA. AT-Cut QCM sensors with a gold electrode, diameter of $6 \mathrm{~mm}$, and resonant frequency of $10 \mathrm{MHz}$ were purchased from Novaetech, Turin, Italy.

The preparation of the electrospinning solution was divided into two steps. First, the PVA solution was prepared by dissolving $1.2 \mathrm{~g}$ PVA powder in $10 \mathrm{~mL}$ distilled water and stirring at a temperature of $95^{\circ} \mathrm{C}$ for $2 \mathrm{~h}$ to obtain a homogenous solution. Second, the PVA solution was mixed with the PEDOT-PSS aqueous dispersion at a ratio of $1: 1(v / v)$. Then, the mixed solution was stirred at ambient temperature for $2 \mathrm{~h}$ to ensure the homogenous dispersion of PEDOT-PSS. The parameters were selected on the basis of the optimization procedure presented in a previous study (Chotimah et al., 2016). Then, the mixed solution was transferred into a $10 \mathrm{~mL}$ plastic syringe for the electrospinning process. The process was conducted in an ambient environment for $30 \mathrm{~s}$ with a high voltage of $10 \mathrm{kV}$ using tip-to-collector distances varying from 7.5 to $12.5 \mathrm{~cm}$. The nanofiber was collected on top of the QCM electrode and dried overnight prior to use. For convenience, the sensor was fabricated using tip-tocollector distances of 7.5, 10, and $12.5 \mathrm{~cm}$, indicated as QCM NF1, QCM NF 2, and QCM NF 3, respectively. The detailed electrospinning parameters used with respect to the produced sensor names are listed in Table 1. The schematic of the electrospinning setup and the chemical structure of the material used are shown in Fig. 1. The morphology of the nanofiber samples was analyzed using a scanning electron microscope (SEM JEOL JSM-6510).

The schematic illustration of the experimental setup used in the humidity test is shown in Fig. 2 and similar to that reported previously (Hidayat et al., 2017). Three types of QCM coated with nanofiber were placed at the top part inside the sensing chamber. To monitor the humidity level inside the chamber, we used the calibrated humidity and temperature sensor SHT31. The humidity variation process is similar to that reported previously (Hidayat et al., 2017). Moreover, the frequency resonance and frequency shift of the QCM sensor were measured using a laboratory-made frequency counter, as reported previously (Rianjanu et al., 2018a; Triyana et al., 2018). 


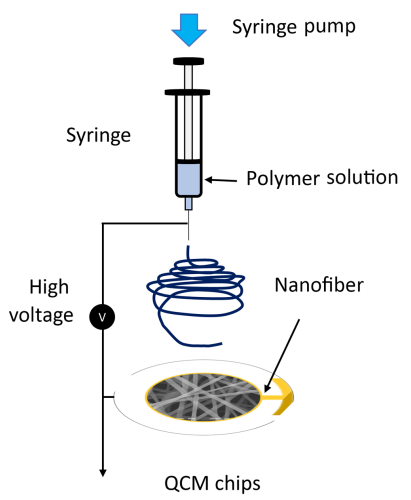

(a)

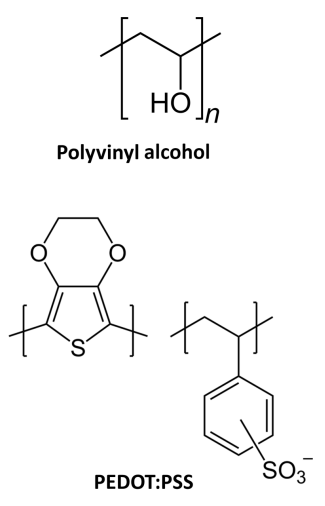

(b)
Figure 1. (a) Electrospinning setup and (b) chemical structure of PVA and PEDOT-PSS.

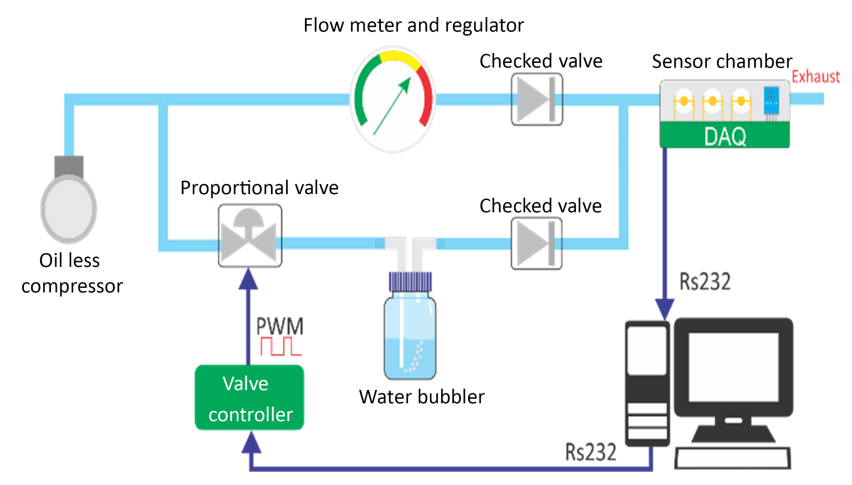

Figure 2. Schematic of the QCM humidity sensing configuration.

\section{Results and discussion}

\subsection{Morphology analysis of the PEDOT-PSS/PVA nanofiber}

Figure 3 shows the SEM image of the PEDOT-PSS/PVA nanofiber sensor. The figure shows that the electrospun nanofiber exhibits a bead-on-string structure for all samples. The bead-on-string structure of the nanofiber can be attributed to the low viscosity of the electrospinning solution. QCM NF 1 and QCM NF 3 show more beads than QCM NF 2. The increase in the number of beads in a nanofiber structure leads to the decrease in its active surface area (Jia et al., 2017) and sensing sensitivity. The 3-D nature of the nanofiber sensor helps facilitate easy contact with water molecules, providing a good candidate for application as a sensitive humidity sensor based on the QCM.

The mass deposited $(\Delta m)$ on top of the QCM surface is strongly related to its frequency shift $(\Delta f)$ calculated using the Sauerbrey equation (Sauerbrey, 1959) and expressed as follows:

$$
\Delta f=-\frac{2 f_{0}^{2}}{A \sqrt{\mu_{q} \rho_{q}}} \Delta m
$$
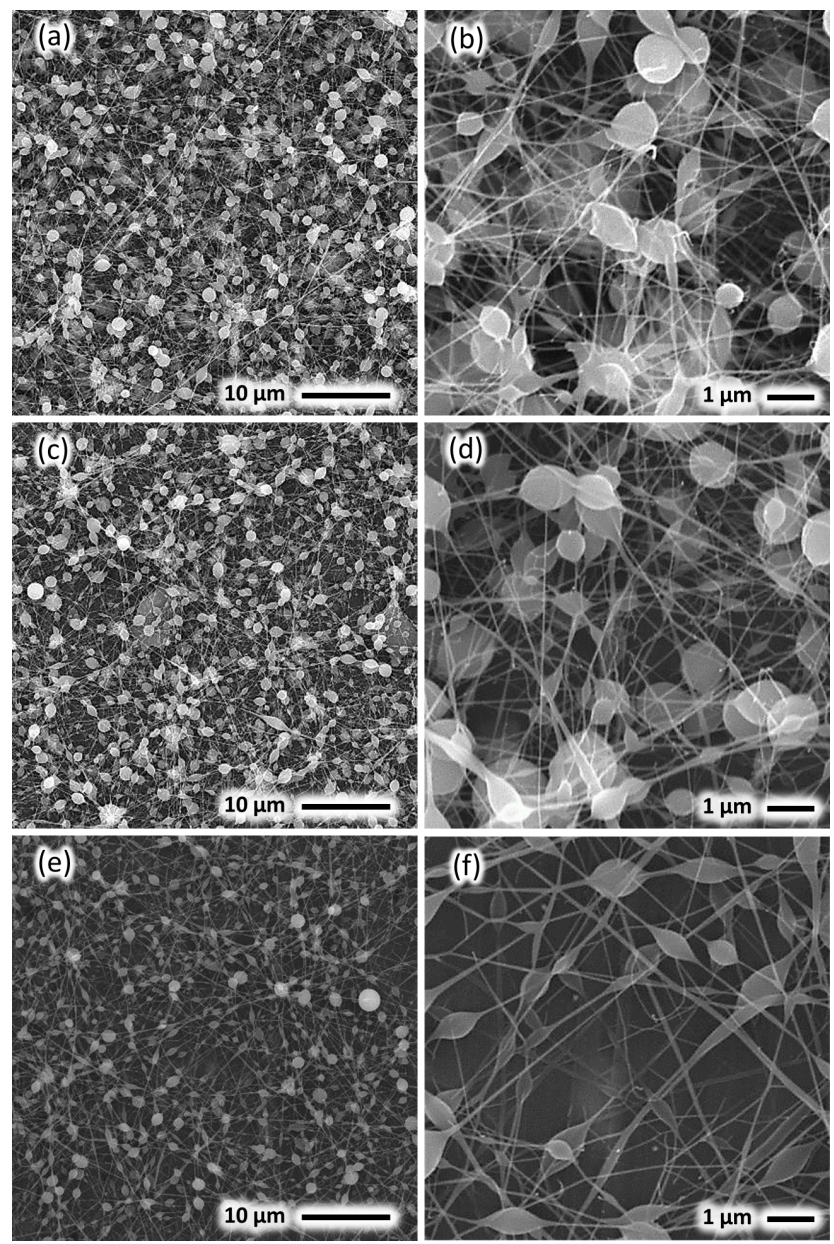

Figure 3. SEM image of the PEDOT-PSS/PVA nanofiber QCM with different magnifications: (a, b) QCM NF 1, (c, d) QCM NF 2, and (e, f) QCM NF 3.

where $f_{0}$ is the frequency base of the $\mathrm{QCM}(\mathrm{Hz}), A$ is the active crystal area $\left(\mathrm{cm}^{3}\right), \mu_{q}$ is the shear modulus $\left(2.95 \times 10^{11} \mathrm{~g} \mathrm{~cm}^{-1} \mathrm{~s}^{-2}\right)$, and $\rho_{Q}$ is the density of the crystal $\left(2.65 \mathrm{~g} \mathrm{~cm}^{-3}\right)$ (Sauerbrey, 1959). The frequency resonance of the QCM before and after deposition was measured at a low humidity level ( $8 \%$ relative humidity $[\mathrm{RH}])$. The frequency shift and calculated loading mass deposition on the QCM surface are shown in Table 1. The table shows that the loading mass decreased with the increase in the tip-to-collector distance. When the deposition rate of the nanofiber process was maintained at a certain level, the amount of nanofiber deposited on the collector depends on the distance and deposition time. At a deposition time of $30 \mathrm{~s}$, the distance affects the mass deposition. The longer the distance is, the less nanofibers are produced.

\subsection{Humidity sensing profile}

Figure 4a shows the frequency response of the QCM nanofiber sensor under the influence of the increasing hu- 

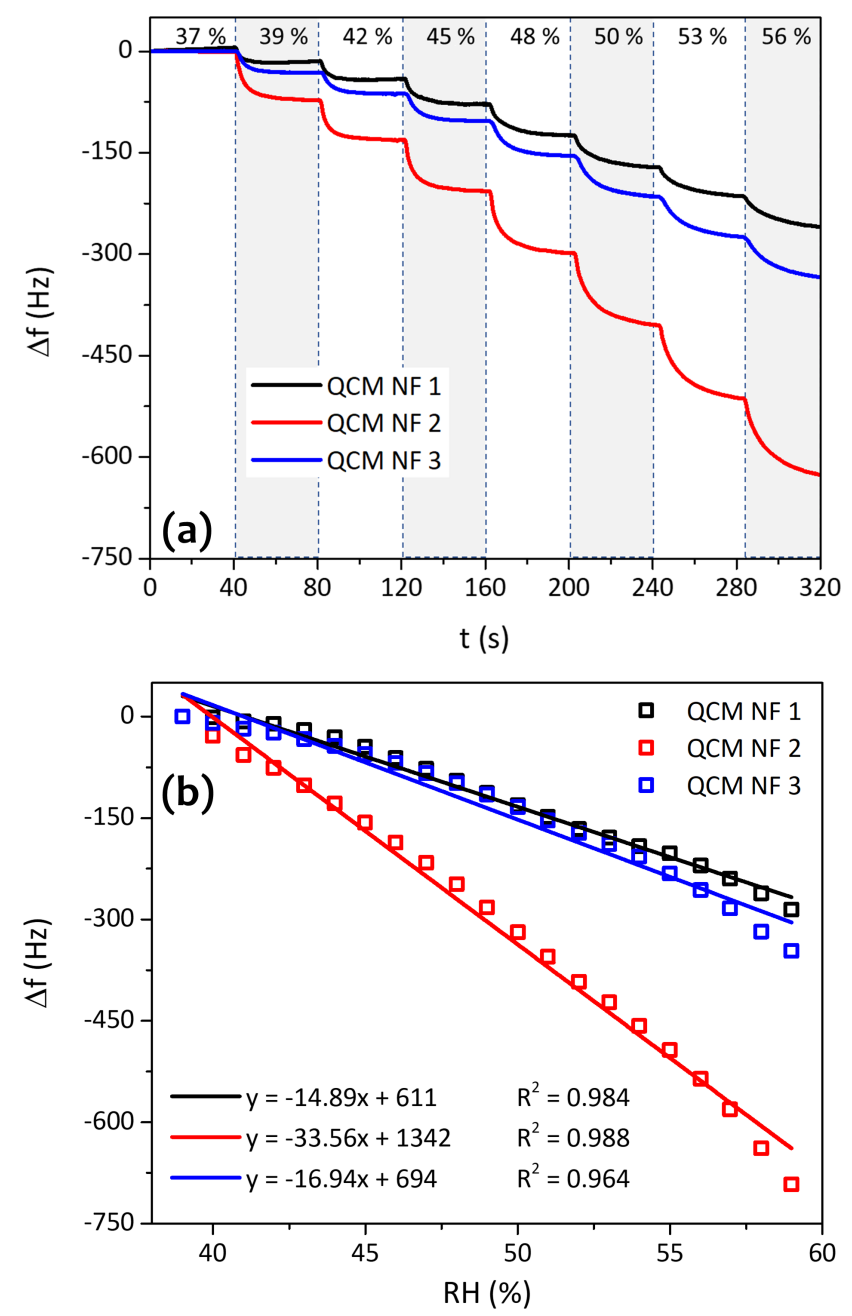

Figure 4. (a) The PEDOT-PSS/PVA QCM response to the increasing humidity level and (b) $\Delta f$ value of the QCM sensor exposed to different humidity levels.

midity level. The frequency response increased with the increasing humidity level of all sensor samples, with the highest response exhibited by the QCM NF 2. The increasing humidity level indicates that more water molecules exist in the surroundings that can interact with and be absorbed into the sensing chamber. The QCM NF 2 sensor can detect a change of the resonance frequency up to $600 \mathrm{~Hz}$ when the humidity level shifts by $19 \%$ (from $37 \%$ to $56 \% \mathrm{RH}$ ), whereas the QCM NF 1 and QCM NF 3 sensors can only detect frequency shifts for 260 and $330 \mathrm{~Hz}$, respectively.

Figure $4 \mathrm{~b}$ shows the sensitivity of the PEDOT-PSS/PVA nanofiber sensor to the change of the humidity level. The data points in Fig. 4b were taken at the steady-state level of QCM response (after $30 \mathrm{~s}$ from the switch on). The sensitivity of the analytical characteristics was determined as the slope of the linear fit of the curve shown in Fig. 4b. The sensitivity value was determined to be $-15,-35$, and $-17 \mathrm{~Hz} \% \mathrm{RH}^{-1}$ for QCM NF 1, QCM NF 2, and QCM NF 3, respectively.
Moreover, the determination coefficient $\left(R^{2}\right)$ was determined to exceed 0.964 , indicating good sensor linearity. This result indicated excellent sensitivity compared with the results of the previous studies (see Table 2). The good sensitivity of the sensor can be attributed to the high surface area of the nanofiber, as mentioned previously.

Other works also noted that the sensitivity of the QCM humidity sensor was proportional to the loading mass of its active layer (Wang et al., 2010). However, this result is different from ours, as the highest sensitivity was exhibited by the QCM NF 2, which has medium loading mass. The QCM NF 1 sample has the highest loading mass, followed by the QCM NF 2 and QCM NF 3 samples. However, the highest sensitivity was exhibited by the QCM NF 2 . We speculated that this phenomenon could be attributed to the higher surface area of the QCM NF 2 sample than that of the other samples. As pointed out in the previous study (Jia et al., 2017), the morphologies of PEDOT-PSS/PVA nanofiber influence its SSA, which in turn affects the sensitivity of the sensor. Thus, it must be noted that the sensitivity of the sensor depends not only on the mass deposition but also on the SSA.

Both response and recovery characteristics are some of the most important parameters in determining the performance of a humidity sensor. A fully dynamic response (responserecovery) of the nanofiber humidity sensor is shown in Fig. 5. The response was recorded during exposure to a low humidity level (32\%) and a high humidity level (67\%). The figure shows that a complete response (absorption) and recovery (desorption) process was performed during the measurement. Moreover, the response time, which is defined as the response value equaling $90 \%$ of the maximum response $\left(t_{90}\right)$, was achieved within 8.8, 5.9, and 5.6 s for QCM NF 1, QCM NF 2, and QCM NF 3, respectively. Meanwhile, the recovery time of the sensor was obtained within 4.0, 3.5, and 3.9s, for QCM NF 1, QCM NF 2, and QCM NF 3, respectively. Both response and recovery times of the PEDOTPSS/PVA nanofiber sensor were faster than those reported in other studies (Table 2). The rapid response and recovery time can be attributed to the rapid absorption of water molecules by the porous structure of nanofiber.

Repeatability is one of the important dynamic characteristics of a humidity sensor. To investigate the repeatability of the PEDOT-PSS/PVA sensor toward the change of the humidity level, the sensor was exposed repeatedly to low (40\%) and high $(58 \%)$ humidity levels. Figure 6a shows the QCM humidity sensing repeatability test for 10 consecutive cycles. The figure shows that the frequency of each nanofiber sensor reaches a similar response value after 10 consecutive cycles. Moreover, the frequency also returns to the previous value after purging, indicating an excellent repeatable sensor. The standard deviation for 10 consecutive cycles was determined to be less than $2 \%$, justifying the excellent repeatability. Figure 6 a also illustrates that the PEDOT-PSS/PVA sensor changes rapidly when in contact with water molecules. 
Table 2. Performance comparison of various humidity sensors based on the QCM.

\begin{tabular}{|c|c|c|c|c|c|c|}
\hline Sensing material & Deposition method & $\begin{array}{r}\text { Sensing } \\
\text { range }(\%)\end{array}$ & $\begin{array}{r}\text { Sensitivity } \\
(\mathrm{Hz} \text { per } \\
\text { percentage } \\
\text { of } \mathrm{RH})\end{array}$ & $\begin{array}{r}\text { Response } \\
\text { time (s) }\end{array}$ & $\begin{array}{r}\text { Recovery } \\
\text { time }(\mathrm{s})\end{array}$ & References \\
\hline $\begin{array}{l}\text { Black phosphorus } \\
\text { nanosheet }\end{array}$ & Drop casting & $11-97$ & - & 14 & 10 & Yao et al. (2017) \\
\hline GO thin film & Spin coating & $6-93$ & 22 & 18 & 12 & Yao et al. (2011) \\
\hline PAA/PVA & Electrospun & $20-95$ & 7 & 84 & - & Wang et al. (2010) \\
\hline Chitosan & Drop casting & $11-94$ & 7 & - & - & Havare et al. (2012) \\
\hline $\mathrm{ZnO}$ nanostructure & Thermal evaporation & $5-97$ & 97 & 90 & 180 & Zhang et al. (2005) \\
\hline PANI micro or nanodots & Electrospun & $20-80$ & 3 & 10 & 10 & $\begin{array}{l}\text { Jaruwongrungsee } \\
\text { et al. (2007) }\end{array}$ \\
\hline PEDOT-PSS/PVA & Electrospun & $40-60$ & 35 & 5.9 & 3.5 & This study \\
\hline
\end{tabular}

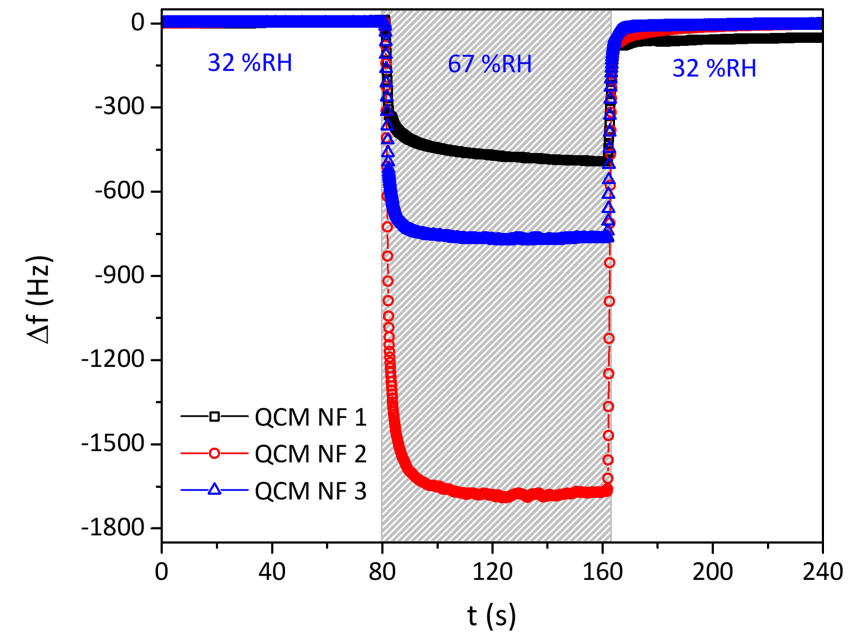

Figure 5. One cycle of the dynamic response of the PEDOTPSS/PVA nanofiber QCM humidity sensor.

Long-term stability is important for the applications of humidity sensors. The frequency response of QCM sensors was regularly tested at $50 \% \mathrm{RH}$ for $16 \mathrm{~d}$ to evaluate their longterm stability. During this period, each QCM sensor was used to measure the humidity level once every $2-3 \mathrm{~d}$. Figure $6 \mathrm{~b}$ displays the frequency shift as a function of time over $16 \mathrm{~d}$ for all sensor samples. The first response is relatively stable during $16 \mathrm{~d}$ of measurement for the QCM chips, indicating the long-term stability of the QCM humidity sensor.

Hysteresis is defined as the noncoincidence relationship between loading and unloading behavior of a sensor. The loading and unloading curves of a perfect sensor normally follow the same path, which could be different for different sensor morphologies. The hysteresis investigation was conducted by measuring the change of the frequency sensor with the consecutively increasing and decreasing humidity levels. Figure 7a shows the frequency shift of QCM NF 2 during the increase and decrease in RH. The figure shows the de-
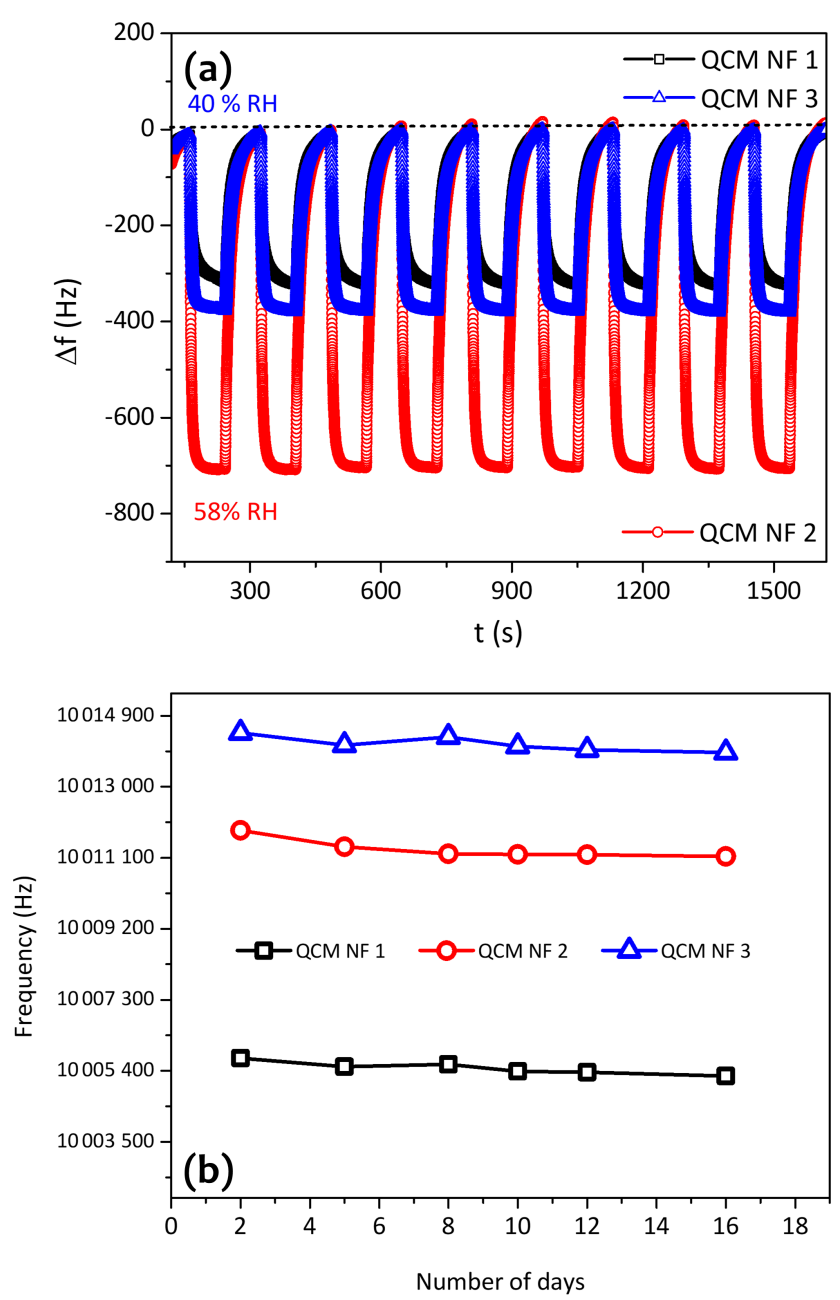

Figure 6. (a) The frequency shift of the PEDOT-PSS/PVA nanofiber sensor during the rapid change of the RH level (low humidity $=40 \%$ to high humidity $=58 \%$ ) for 10 consecutive cycles and (b) long-term stability graph of the PEDOT-PSS/PVA nanofiber-coated QCM humidity sensor. 

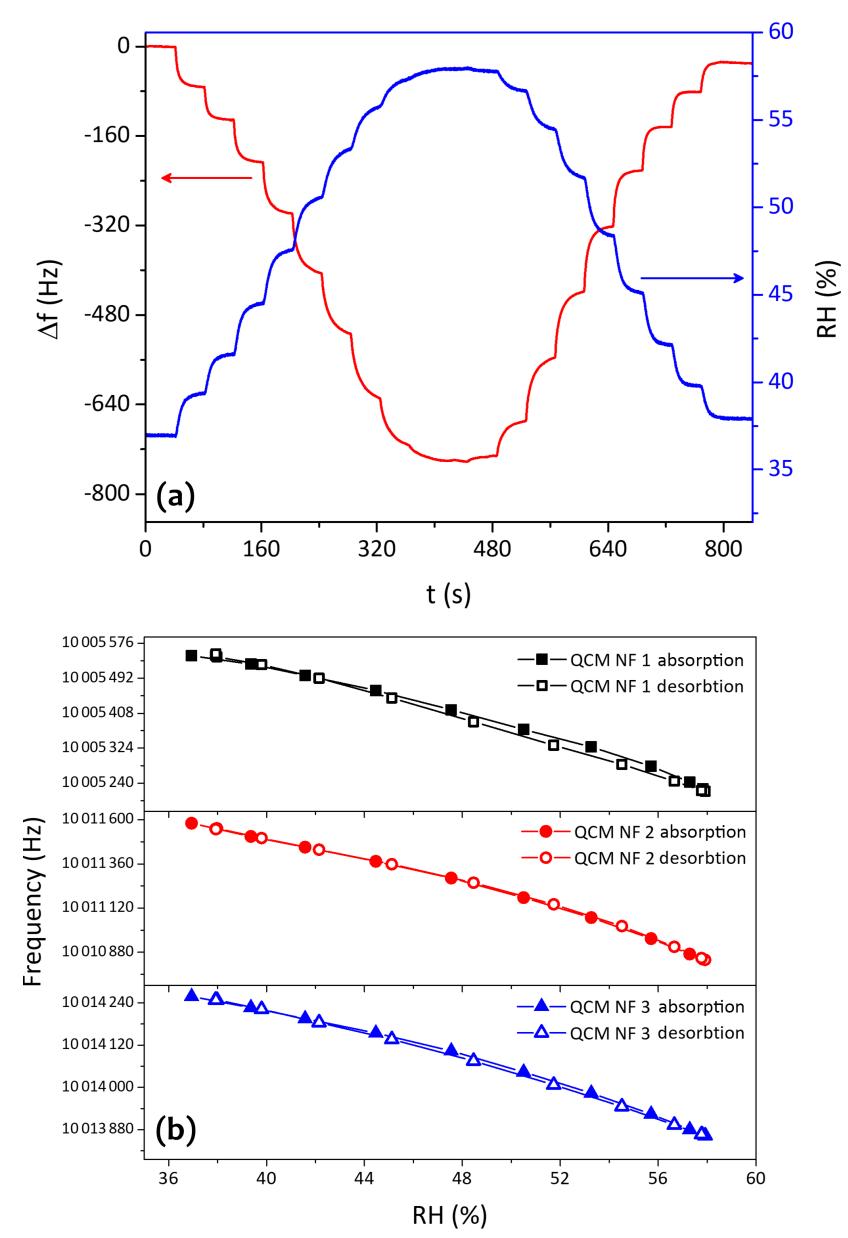

Figure 7. (a) PEDOT-PSS/PVA nanofiber QCM NF 2 humidity sensor $\Delta f$ during the increase and decrease in humidity level and (b) hysteresis profile.

pendency of the frequency response of the QCM nanofiber sensor on the absorption and desorption of water molecules. The steady-state response value of the QCM nanofiber sensor corresponds to its humidity value plotted in the hysteresis graph shown in Fig. 7b. The hysteresis level can be determined using Eq. (2) (Bae et al., 2017), as follows:

hysteresis $=\frac{\left(f_{r}^{\mathrm{a}}-f_{r}^{\mathrm{d}}\right)}{S}$,

where $f_{r}^{\mathrm{a}}$ and $f_{r}^{\mathrm{d}}$ are the frequencies at $r \%$ RH during the absorption (a) and desorption (d) processes, respectively, and $S$ is the sensitivity of the sensor. The hysteresis of QCM NF 1, QCM NF 2, and QCM NF 3 was determined to be $1.3 \%$, $0.25 \%$, and $0.64 \% \mathrm{RH}$, respectively. The lowest hysteresis level was exhibited by the QCM NF 2, which can be attributed to the high porosity of QCM NF 2 . The results indicated that all of the QCM sensors have low hysteresis and high potential for use as a humidity sensor.

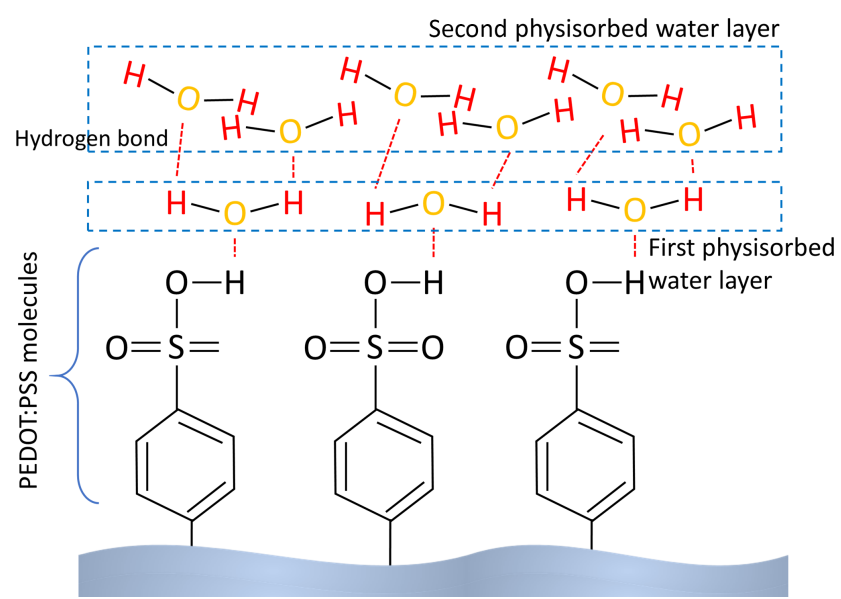

Figure 8. Schematic illustrations of probable interactions between water molecules and PEDOT-PSS sensing layer.

The results indicated that the PEDOT-PSS/PVA QCM nanofiber sensor is sensitive to water molecule adsorption, exhibiting good repeatability and rapid response and recovery times, which can be attributed to the hydrophilic group on PEDOT-PSS. Figure 8 shows the schematic illustration of probable water molecule adsorption at the PEDOT-PSS/PVA nanofiber sensor. As previously investigated (Muckley et al., 2016), the water molecule was adsorbed onto the PSS film through hydrogen bonding with the hydroxyl group of PSS molecules. Hydrogen bonding between adsorbed $\mathrm{H}_{2} \mathrm{O}$ and vapor-phase $\mathrm{H}_{2} \mathrm{O}$ accelerates $\mathrm{H}_{2} \mathrm{O}$ sorption, resulting in the high response of the sensor under a high humidity level.

\section{Conclusions}

This work describes the fabrication and sensor characteristic investigation of QCM coated with PEDOT-PSS/PVA nanofiber as a humidity sensor. The PEDOT-PSS/PVA sensor possessed a bead-on-string structure, as confirmed by the SEM images. The nanofiber sensor exhibits excellent sensitivity, with rapid response and recovery times, good repeatability, long-term stability, and low hysteresis (less than $1.3 \%)$. The highest sensing performance was exhibited by the QCM NF 2. The hydrophilic group on PEDOT-PSS is responsible for the sensing mechanism via hydrogen bonding with the hydroxyl group of PSS molecules. Furthermore, this work shows the potential of PEDOT-PSS/PVA nanofiber for use as a humidity sensor with good sensing performance.

Data availability. Research data are available upon request to the authors.

Author contributions. TJ performed the experiments, analyzed the data, and designed and developed the frequency measurement 
system. AR performed the experiments, analyzed the data, and wrote the initial paper. SNH designed and developed the frequency measurement system and performed the experiments. AK and RR designed the experiments, contributed reagent and materials, analyzed the data, and revised the paper. KT conceived and designed the experiments, revised the paper, and lead the project. All authors approved the final paper.

Competing interests. The authors declare that they have no conflict of interest.

Acknowledgements. We thank Setyawan P. Sakti for characterizing the conductance and susceptance of the QCM using a vector network analyzer (Omicron-Lab Bode 100).

Financial support. This research has been supported by the Ministry of Research, Technology and Higher Education of the Republic of Indonesia under the scheme of Penelitian Terapan (contract no. 2833/UN1.DITLIT/DIT-LIT/LT/2019).

Review statement. This paper was edited by Nobutito Imanaka and reviewed by two anonymous referees.

\section{References}

Adamyan, Z., Sayunts, A., Aroutiounian, V., Khachaturyan, E., Vrnata, M., Fitl, P., and Vlček, J.: Nanocomposite sensors of propylene glycol, dimethylformamide and formaldehyde vapors, J. Sens. Sens. Syst., 7, 31-41, https://doi.org/10.5194/jsss-7-312018, 2018.

Bae, Y. M., Lee, Y. H., Kim, H. S., Lee, D. J., Kim, S. Y., and Kim, H.-D.: Polyimide-polyurethane/urea block copolymers for highly sensitive humidity sensor with low hysteresis, J. Appl. Polym. Sci., 134, 1-11, https://doi.org/10.1002/app.44973, 2017.

Beißner, S., Thies, J.-W., Bechthold, C., Kuhn, P., Thürmann, B., Dübel, S., and Dietzel, A.: Low-cost, in-liquid measuring system using a novel compact oscillation circuit and quartz-crystal microbalances (QCMs) as a versatile biosensor platform, J. Sens. Sens. Syst., 6, 341-350, https://doi.org/10.5194/jsss-6-341-2017, 2017.

Blank, T. A., Eksperiandova, L. P., and Belikov, K. N.: Recent trends of ceramic humidity sensors development: A review, Sensor. Actuat. B-Chem., 228, 416-442, https://doi.org/10.1016/j.snb.2016.01.015, 2016.

Chen, X., Chen, X., Li, N., Ding, X., and Zhao, X.: A QCM Humidity Sensors Based on GO/Nafion Composite Films With Enhanced Sensitivity, IEEE Sens. J., 16, 8874-8883, https://doi.org/10.1109/JSEN.2016.2617122, 2016.

Cho, D., Hoepker, N., and Frey, M. W.: Fabrication and characterization of conducting polyvinyl alcohol nanofibers, Mater. Lett., 68, 293-295, https://doi.org/10.1016/j.matlet.2011.10.109, 2012.

Choi, J., Lee, J., Choi, J., Jung, D., and Shim, S. E.: Electrospun PEDOT:PSS/PVP nanofibers as the chemiresistor in chemical vapour sensing, Synthetic Met., 160, 1415-1421, https://doi.org/10.1016/j.synthmet.2010.04.021, 2010.

Choi, K. H., Sajid, M., Aziz, S., and Yang, B. S.: Wide range high speed relative humidity sensor based on PEDOT:PSS-PVA composite on an IDT printed on piezoelectric substrate, Sensor. Actuator. A-Phys., 228, 40-49, https://doi.org/10.1016/j.sna.2015.03.003, 2015.

Chotimah, C., Rianjanu, A., Winardianto, B., Munir, M., Kartini, I., and Triyana, K.: Electrical Conductivity Improvement of Polyvinyl Alcohol Nanofiber by Solvent Vapour Treatment, Int. J. Adv. Sci. Eng. Inf. Technol., 6, 675, https://doi.org/10.18517/ijaseit.6.5.1055, 2016.

Ding, B., Wang, M., Yu, J., and Sun, G.: Gas sensors based on electrospun nanofibers, Sensors, 9, 1609-1624, https://doi.org/10.3390/s90301609, 2009.

Haidry, A. A., Kind, N., and Saruhan, B.: Investigating the influence of Al-doping and background humidity on $\mathrm{NO}_{2}$ sensing characteristics of magnetron-sputtered $\mathrm{SnO}_{2}$ sensors, J. Sens. Sens. Syst., 4, 271-280, https://doi.org/10.5194/jsss-4-271-2015, 2015.

Havare, A. K., Ilgu, H., Okur, S., and Sanli-Mohamed, G.: Humidity Sensing Properties of Chitosan by Using Quartz Crystal Microbalance Method, Sens. Lett., 10, 906-910, https://doi.org/10.1166/s1.2012.2585, 2012.

Hidayat, S. N., Julian, T., Rianjanu, A., Kusumaatmadja, A., Triyana, K., and Roto, R.: Quartz crystal microbalance coated by PAN nanofibers and PEDOT:PSS for humidity sensor, in: 2017 International Seminar on Sensors, Instrumentation, Measurement and Metrology (ISSIMM), 2017, 119-123, IEEE, 2017.

Hossein-Babaei, F., Akbari, T., and Harkinezhad, B.: Dopant passivation by adsorbed water monomers causes high humidity sensitivity in PEDOT: PSS thin films at ppmlevel humidity, Sensor. Actuator. B-Chem., 293, 329-335, https://doi.org/10.1016/j.snb.2019.05.018, 2019.

Jaruwongrungsee, K., Tuantranont, A., Wanna, Y., Wisitsoraat, A., and Lomas, T.: Quartz Crystal Microbalance humidity sensor using electrospun PANI micro/nano dots, in: 2007 7th IEEE Conference on Nanotechnology (IEEE NANO), 316-319, IEEE, 2007.

Jaruwongrungsee, K., Sriprachuabwong, C., Sappat, A., Wisitsoraat, A., Phasukkit, P., Sangworasil, M., and Tuantranont, A.: High-sensitivity humidity sensor utilizing PEDOT/PSS printed quartz crystal microbalance, in: The 8th Electrical Engineering/ Electronics, Computer, Telecommunications and Information Technology (ECTI) Association of Thailand - Conference 2011, 66-69, IEEE, 2011.

Jia, Y., Yu, H., Cai, J., Li, Z., and Dong, F.: Explore on the quantitative analysis of specific surface area on sensitivity of polyacrylic acid-based QCM ammonia sensor, Sensor. Actuator. B-Chem., 243, 1042-1045, https://doi.org/10.1016/j.snb.2016.12.090, 2017.

Jin, H., Tao, X., Feng, B., Yu, L., Wang, D., Dong, S., and Luo, J.: A humidity sensor based on quartz crystal microbalance using graphene oxide as a sensitive layer, Vacuum, 140, 101-105, https://doi.org/10.1016/j.vacuum.2016.10.017, 2017.

Kumar, S., Rai, P., Sharma, J. G., Sharma, A., and Malhotra, B. D.: PEDOT:PSS/PVA-Nanofibers-Decorated Conducting Paper for Cancer Diagnostics, Adv. Mater. Technol., 1, 1600056, https://doi.org/10.1002/admt.201600056, 2016. 
Lensch, H., Bastuck, M., Baur, T., Schütze, A., and Sauerwald, T.: Impedance model for a high-temperature ceramic humidity sensor, J. Sens. Sens. Syst., 8, 161-169, https://doi.org/10.5194/jsss8-161-2019, 2019.

Li, J., Kaku, T., Tokura, Y., Matsukawa, K., Homma, K., Nishimoto, T., Hiruta, Y., Akimoto, A. M., Nagase, K., Kanazawa, H., and Shiratori, S.: Adsorption-Desorption Control of Fibronectin in Real Time at the Liquid/Polymer Interface on a Quartz Crystal Microbalance by Thermoresponsivity, Biomacromolecules, 20, 1748-1755, https://doi.org/10.1021/acs.biomac.9b00121, 2019.

Liu, N., Fang, G., Wan, J., Zhou, H., Long, H., and Zhao, X.: Electrospun PEDOT:PSS-PVA nanofiber based ultrahigh-strain sensors with controllable electrical conductivity, J. Mater. Chem., 21, 18962, https://doi.org/10.1039/c1jm14491j, 2011.

Muckley, E. S., Lynch, J., Kumar, R., Sumpter, B. and Ivanov, I. N.: PEDOT:PSS/QCM-Based Multimodal Humidity and Pressure Sensor, Sensor. Actuator. B-Chem., 236, 91-98, https://doi.org/10.1016/j.snb.2016.05.054, 2016.

Muckley, E. S., Jacobs, C. B., Vidal, K., Mahalik, J. P., Kumar, R., Sumpter, B. G., and Ivanov, I. N.: New Insights on Electro-Optical Response of Poly(3,4ethylenedioxythiophene):Poly(styrenesulfonate) Film to Humidity, ACS Appl. Mater. Inter., 9, 15880-15886, https://doi.org/10.1021/acsami.7b03128, 2017.

Nie, J., Liu, J., Li, N., and Meng, X.: Dew point measurement using dual quartz crystal resonator sensor, Sensor. Actuator. B-Chem., 246, 792-799, https://doi.org/10.1016/j.snb.2017.02.166, 2017.

Park, J.-K., Kang, T.-G., Kim, B.-H., Lee, H.-J., Choi, H. H., and Yook, J.-G.: Real-time Humidity Sensor Based on Microwave Resonator Coupled with PEDOT:PSS Conducting Polymer Film, Sci. Rep., 8, 439, https://doi.org/10.1038/s41598-017-18979-3, 2018.

Pascariu, P., Airinei, A., Olaru, N., Petrila, I., Nica, V., Sacarescu, L., and Tudorache, F.: Microstructure, electrical and humidity sensor properties of electrospun NiO$\mathrm{SnO} 2$ nanofibers, Sensor. Actuator. B-Chem., 222, 1024-1031, https://doi.org/10.1016/j.snb.2015.09.051, 2016.

Rianjanu, A., Roto, R., Julian, T., Hidayat, S., Kusumaatmaja, A., Suyono, E., and Triyana, K.: Polyacrylonitrile Nanofiber-Based Quartz Crystal Microbalance for Sensitive Detection of Safrole, Sensors, 18, 1150, https://doi.org/10.3390/s18041150, 2018a.

Rianjanu, A., Julian, T., Hidayat, S. N., Suyono, E. A., Kusumaatmaja, A., and Triyana, K.: Polyacrylonitrile nanofiber as polar solvent $\mathrm{N}, \mathrm{N}$-dimethyl formamide sensor based on quartz crystal microbalance technique, J. Phys. Conf. Ser., 1011, 012067, https://doi.org/10.1088/1742-6596/1011/1/012067, 2018b.

Rianjanu, A., Kusumaatmaja, A., Suyono, E. A., and Triyana, K.: Solvent vapor treatment improves mechanical strength of electrospun polyvinyl alcohol nanofibers, Heliyon, 4, e00592, https://doi.org/10.1016/j.heliyon.2018.e00592, 2018 c.

Rianjanu, A., Hidayat, S. N., Julian, T., Suyono, E. A., Kusumaatmaja, A., and Triyana, K.: Swelling Behavior in Solvent Vapor Sensing based on Quartz Crystal Microbalance (QCM) Coated Polyacrylonitrile (PAN) Nanofiber, IOP Conf. Ser. Mater. Sci. Eng., 367, 012020, https://doi.org/10.1088/1757899X/367/1/012020, 2018d.

Rianjanu, A., Hasanah, S. A., Nugroho, D. B., Kusumaatmaja, A., Roto, R., and Triyana, T.: Polyvinyl Acetate Film-Based Quartz Crystal Microbalance for the Detection of Benzene,
Toluene, and Xylene Vapors in Air, Chemosensors, 7, 20, https://doi.org/10.3390/chemosensors7020020, 2019.

Rodoplu, D. and Mutlu, M.: Effects of electrospinning setup and process parameters on nanofiber morphology intended for the modification of quartz crystal microbalance surfaces, J. Eng. Fiber. Fabr., 7, 118-123, 2012.

Sauerbrey, G.: Verwendung von Schwingquarzen zur Wägung dünner Schichten und zur Mikrowägung, Z. Phys., 155, 206-222, https://doi.org/10.1007/BF01337937, 1959.

Taccola, S., Greco, F., Zucca, A., Innocenti, C., de Julián Fernández, C., Campo, G., Sangregorio, C., Mazzolai, B., and Mattoli, V.: Characterization of Free-Standing PEDOT:PSS/Iron Oxide Nanoparticle Composite Thin Films and Application As Conformable Humidity Sensors, ACS Appl. Mater. Inter., 5, 63246332, https://doi.org/10.1021/am4013775, 2013.

Triyana, K., Sembiring, A., Rianjanu, A., Hidayat, S., Riowirawan, R., Julian, T., Kusumaatmaja, A., Santoso, I., and Roto, R.: Chitosan-Based Quartz Crystal Microbalance for Alcohol Sensing, Electronics, 7, 181, https://doi.org/10.3390/electronics7090181, 2018.

Wang, X., Ding, B., Yu, J., Wang, M., and Pan, F.: A highly sensitive humidity sensor based on a nanofibrous membrane coated quartz crystal microbalance, Nanotechnology, 21, 055502, https://doi.org/10.1088/0957-4484/21/5/055502, 2010.

Xu, J., Wang, L., Cha, X., Wu, Y., Xu, J., Cheng, Z., and Xiang, Q.: Superhydrophobic Polymerized n-Octadecylsilane Surface for BTEX Sensing and Stable Toluene/Water Selective Detection Based on QCM Sensor, ACS Omega, 3, 2437-2443, https://doi.org/10.1021/acsomega.8b00061, 2018.

Xu, J., Bertke, M., Wasisto, H. S., and Peiner, E.: Piezoresistive microcantilevers for humidity sensing, J. Micromech. Microeng., 29, 053003, https://doi.org/10.1088/1361-6439/ab0cf5, 2019.

Yang, J., Xu, J., Wu, W., Bertke, M., Wasisto, H. S., and Peiner, E.: Piezoresistive Silicon Cantilever Covered by $\mathrm{ZnO}$ Nanorods for Humidity Sensing, Procedia Eng., 168, 1114-1117, https://doi.org/10.1016/j.proeng.2016.11.361, 2016.

Yao, Y., Chen, X., Guo, H., and Wu, Z.: Graphene oxide thin film coated quartz crystal microbalance for humidity detection, Appl. Surf. Sci., 257, 7778-7782, https://doi.org/10.1016/j.apsusc.2011.04.028, 2011.

Yao, Y., Zhang, H., Sun, J., Ma, W., Li, L., Li, W., and Du, J.: Novel QCM humidity sensors using stacked black phosphorus nanosheets as sensing film, Sensor. Actuator. B-Chem., 244, 259-264, https://doi.org/10.1016/j.snb.2017.01.010, 2017.

Zhang, D., Wang, D., Li, P., Zhou, X., Zong, X., and Dong, G.: Facile fabrication of high-performance QCM humidity sensor based on layer-by-layer self-assembled polyaniline/graphene oxide nanocomposite film, Sensor. Actuator. B-Chem., 255, 18691877, https://doi.org/10.1016/j.snb.2017.08.212, 2018.

Zhang, Y., Yu, K., Ouyang, S., Luo, L., Hu, H., Zhang, Q., and Zhu, Z.: Detection of humidity based on quartz crystal microbalance coated with $\mathrm{ZnO}$ nanostructure films, Phys. B Condens. Matter, 368, 94-99, https://doi.org/10.1016/j.physb.2005.07.001, 2005. 